\title{
The Potential of Multilayered Hierarchical Nonterrestrial Networks for 6G: A Comparative Analysis Among Networking Architectures
}

\author{
Dengke Wang, Student Member, IEEE, Marco Giordani, Member, IEEE, \\ Mohamed-Slim Alouini, Fellow, IEEE, and Michele Zorzi, Fellow, IEEE
}

\begin{abstract}
G) communication research is currently focusing on non-terrestrial networks (NTNs) to promote ubiquitous and ultra-high-capacity global connectivity. Specifically, multi-layered hierarchical networks, i.e., the orchestration among different aerial/space platforms, including Low and High Altitude Platforms (LAPs and HAPs), and satellites co-operating at different altitudes, currently represent one the most attractive technological options to solve coverage and latency constraints associated with the NTN paradigm. However, there are still several issues to be resolved for proper network design. In this work, we evaluate the performance of different multi-layered non-terrestrial configurations, and provide guidelines on the optimal working point(s) for which it is possible to achieve a good compromise between improved system flexibility and network performance, with respect to a baseline standalone deployment.
\end{abstract}

Index Terms-6G, non-terrestrial networks (NTNs), HAPs, satellites, multi-layered networks.

\section{INTRODUCTION}

With 5th generation (5G) wireless networks ready for commercial roll-out, 6 th generation $(6 \mathrm{G})$ research $[1]$ is currently concentrating on the development of non-terrestrial networks (NTNs) in which Low Altitude Platforms (LAPs), High Altitude Platforms (HAPs), and satellites expand traditional bi-dimensional network designs to operate in the three-dimensional space [2]. In particular, air/spaceborne stations can assist terrestrial infrastructures in promoting flexible global connectivity in crowded areas, cost-effective network coverage in public safety situations, and last-mile service delivery and backhaul in remote/rural/hard-to-access zones [3]. In this perspective, the 3rd Generation Partnership Project (3GPP) has approved the first Rel-17 specifications to support NTNs in 5G NR systems, and study items are encouraged for Rel-18 and Rel-19, thus acknowledging long-term research towards 6G [4].

Until the advent of $5 \mathrm{G}, \mathrm{NTN}$ have been mainly relegated to support services like television broadcasting, meteorology, and navigation, which are generally provided by standalone satellite constellations operating on Low Earth Orbits (LEOs) or Geostationary Earth Orbits (GEOs). On one side, satellites offer a number of advantages to ground users, including

D. Wang and M.-S. Alouini are with the King Abdullah University of Science and Technology (KAUST), Thuwal, Saudi Arabia (email: \{dengke.wang,slim.alouini\}@kaust.edu.sa). M. Giordani (corresponding author) and M. Zorzi are with the Department of Information Engineering (DEI), University of Padova, Italy (email: \{giordani,zorzi\} @ dei.unipd.it). extremely large coverage regions and favorable line-of-sight connectivity. On the other side, satellite deployments typically suffer from severe path loss, huge communication delays, and expensive installation costs [5]. These weaknesses can be efficiently downplayed by complementing satellite systems with the participation of HAPs and LAPs. In addition to their versatility and lower cost, these elements achieve lower latency thanks to the shorter distances involved, and can operate with spot beams delivering more capacity to ground users, more diversity options, or a combination of the two. However, not only do LAPs, specifically Unmanned Aerial Vehicles (UAVs), incur significant energy consumption for propulsion and hovering, but also the intrinsic mobility of HAPs could impair network performance without proper coordination and countermeasures. In this context, interconnecting space, air, and ground networks is emerging as a viable approach to enhance communication, with each network segment compensating for the weaknesses of the other.

Initial studies have demonstrated that the availability of multi-layered hierarchical networks can provide better coverage, resilience, and flexibility compared to standalone deployments, which makes them suitable for several practical fields in future networks, including traffic control and emergency communication [6]. Software-defined networks may also offer a programmable, scalable, and customizable framework to integrate space, air, and ground components for matching traffic demands with the available network capacity [7]. In recent years, private organizations have also financed projects to provide broadband internet to the world by combining the persistence of satellites and HAPs with the flexibility of UAVs, such as Airbus Zephyr's initiative. However, unlike traditional standalone architectures, multi-layered NTNs are affected by limitations related to traffic distribution, resource allocation, load balancing, and mobility management, which require end-to-end (rather than point-to-point) optimization. In this context, there are still various questions to be answered for proper network design, in particular which degree of integration results in better spatio-temporal coverage.

Along these lines, in this work we evaluate the performance of different hierarchical NTN architectures in various scenarios, and provide guidelines on the working point(s) that achieve a good compromise between system flexibility and network capacity, against a baseline standalone GEO deployment. Unlike prior works, which typically consider hybrid satellite terrestrial networks only, we study whether a fully 


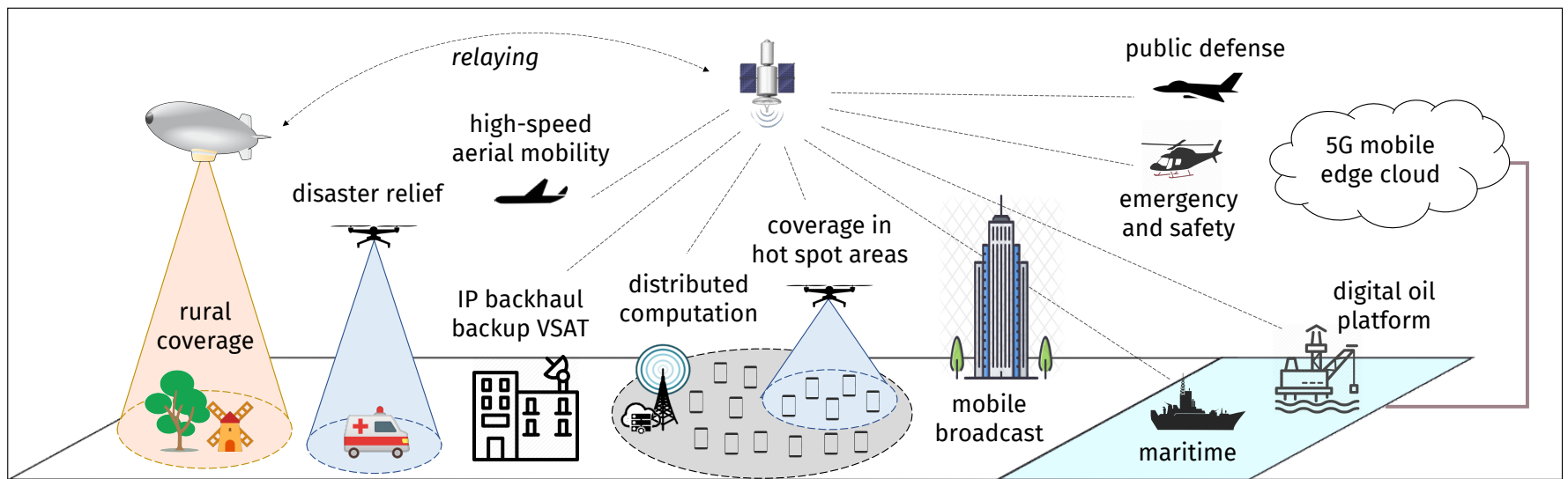

Fig. 1: Use cases enabled by non-terrestrial networks.

integrated space-air-ground architecture may result in better coverage. Moreover, we characterize which combinations of parameters, including the operational frequency, the deployment altitude, the antenna configuration, and the quality of service requirements, result in optimized network performance. To this aim, we adopt the architectural designs and related system parameters provided by the $3 \mathrm{GPP}$ and the International Telecommunication Union (ITU) technical reports for NTNs, thus guaranteeing accurate and realistic system-level analyses. Our results demonstrate that, while GEO satellite operations can be improved by deploying intermediate stratospheric HAPs, power/antenna constraints on spaceborne vehicles make it undesirable to adopt LEO satellites to relay the upstream GEO signals.

The remainder of this paper is organized as follows. In Sec. II we discuss the main NTN use cases and possible methods to cooperatively integrate non-terrestrial layers, in Sec. III we describe our system model, and in Sec. IV we present our main findings and simulation results. Concluding remarks are finally provided in Sec. V

\section{Multi-Layered Non-Terrestrial Networks}

NTNs play a leading role in $5 \mathrm{G}$ and beyond by covering different verticals, including health care, intelligent transportation, public safety, and many others. In these regards, multi-layered NTNs can further exploit the complementary advantages of space, air, and ground facilities to make the best use of the 3D paradigm. In Sec. II-A we review potential NTN use cases, while in Sec. II-B, we present possible solutions to integrate non-terrestrial layers.

\section{A. NTN Use Cases}

Recent technological innovations in the aerial/space industry have made it possible to enable advanced use cases for NTNs [8], [9], as illustrated in Fig. 1] and discussed below.

- Service Continuity, i.e., providing connectivity when terrestrial networks are overloaded (e.g., in hot spot areas or during rush hours) or in those regions where installation of terrestrial infrastructure is too expensive or even impossible (e.g., above oceans or deserts). NTNs can also improve communication availability in remote or disaster zones, or to preserve the connection in emergency situations when the primary terrestrial path is out of service. For these types of services, reliability is a critical requirement: the interruption time for data packet exchange should be maintained below $1 \mathrm{~ms}$.

- Service Ubiquity, i.e., guaranteeing global umbrella coverage via aerial/space links. The wide broadcast nature of non-terrestrial platforms can indeed support multimedia content provisioning to wide geographical areas, and serve wireless backhaul traffic requests in rural locations with no fiber backhaul solutions. In the next 10 years, NTNs are expected to provide a low-latency connection of at least $10 \mathrm{Mbps}$ in rural/remote areas, and a $75 \%$ Internet penetration worldwide.

- Service Scalability, i.e., offloading traffic from terrestrial networks to more computationally powerful space/air nodes for timely (and aggregated) processing of data, while allowing simplified hardware design on the ground. NTNs also promote energy sustainability as aerial platforms, operated through renewable sources, can be deployed on demand, unlike always-on terrestrial stations.

\section{B. Integrating Non-Terrestrial Layers}

GEO satellites, with their coverage umbrella of around $7,300 \mathrm{~km}$ of radius, can provide stable and cost-effective connection for broadcast services. However, the GEO channel suffers from a tremendous propagation delay of around 240 $\mathrm{ms}$ and severe path loss due to atmospheric absorption over great distances [5], which make point-to-point GEO deployments (Configuration GE in Fig. 2) not desirable for 5G/6G applications. Collaboration among aerial/space platforms has thus been proposed to improve network performance. While LAP/UAV-assisted networks are foreseen (e.g., to enhance public safety and support traffic requirements in hot-spot areas [10], as illustrated in Fig. 1), we focus on satellite- and HAP-based deployments, which do not pose power/mobility constraints and are becoming a hot research topic.

GEO-LEO Integration (Configuration GLE in Fig. 2). The introduction of frequent and affordable orbital insertions in the Low Earth Orbit (typically between 600 and 1,500 km) opens up new opportunities for communication. Compared to 


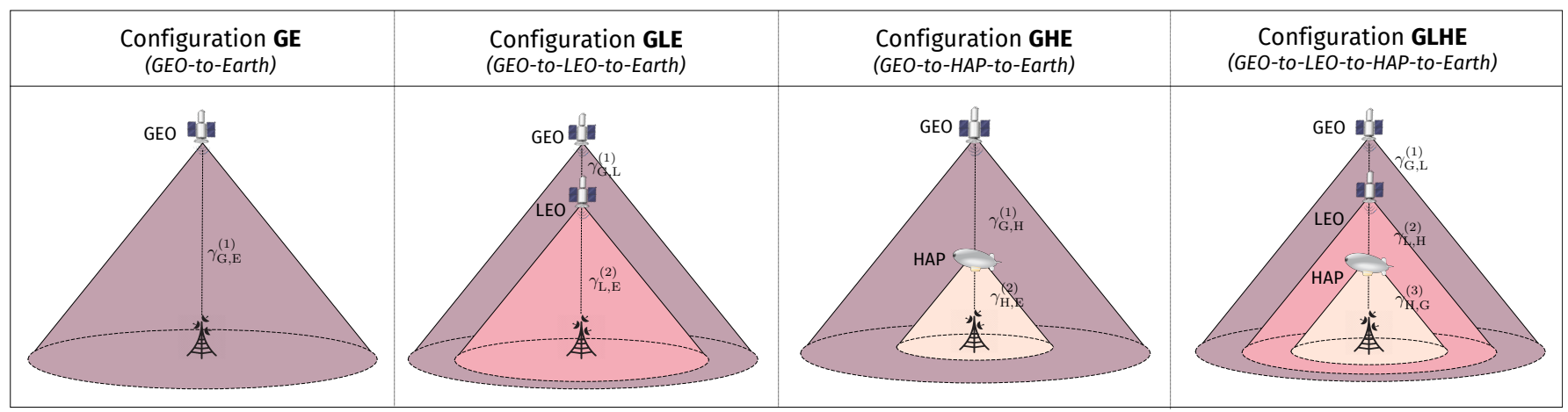

Fig. 2: Possible multi-layered NTN configurations based on a GEO satellite. In Configuration GE direct GEO-ground communication is involved; in Configuration GLE (GHE) a LEO (HAP) bridges the GEO communications towards the ground; in Configuration GLHE a complete integrated space-air-ground architecture is considered.

standalone GEO constellations, the GLE framework improves optical sensors resolution and geographical position accuracy thanks to the increased proximity to the targets, thus offering the same sensing efficiency with lighter payloads and smaller size or, equivalently, incorporating more capable platforms at the same cost. Moreover, it guarantees improved payload performance since LEO satellites, which operate in rarified environments, can implement aerodynamic deorbiting and maneuvers via air breathing electric propulsion. Furthermore, GLE achieves better wireless coverage and lower latency since LEOs can amplify and relay the upstream GEO signals towards the ground. GLE also provides both dense and comprehensive coverage when GEO's ability to support connectivity over very large areas while being continuously visible from terrestrial terminals and LEO's flexibility are leveraged together.

However, the high-speed mobility and related Doppler shift experienced in the lower orbits require dense constellations of satellites to maintain signal continuity on Earth. A standalone space network may also complicate delay-sensitive delivery of data as the channel capacity has to be split among a very large number of ground terminals, thus saturating the available bandwidth.

GEO-HAP Integration (Configuration GHE in Fig. 2). HAPs, which operate in the stratosphere, can act as wireless relays to improve global connectivity, as acknowledged in the literature [11]. Compared to the GE and GLE configurations, GHE not only improves capacity by amplifying the GEO signal before forwarding it to the ground, but also ensures quicker and cheaper deployment. Also, it offers adaptive networking capabilities as the network topology can be adjusted on demand based on instantaneous temporal and traffic demands. The GHE approach guarantees continuous end-to-end coverage as HAPs, unlike LEO satellites, operate in a quasistationary position, and allow communication equipment to operate with less interference and/or distortion. Additionally, GHE permits to host computing and storage facilities on HAPs, i.e., closer to the ground users, rather than on satellites, thus promoting better latency and reliability for applications like mission offloading thanks to the more favorable link budget in the HAP-Earth link. However, while standalone GEO deployments can offer wide geographical connectivity, an intermediate HAP will necessarily reduce the footprint shaped on the ground, and should operate in constellations/swarms to ensure seamless coverage.

GEO-LEO-HAP Integration (Configuration GLHE in Fig. 2). A three-hop integrated network can further enhance communication performance by building a seamless reconfigurable network environment that provides a much larger coverage than a classic terrestrial network [6]. The upper layer is a bi-dimensional satellite network, which may be organized in a mesh topology to create an overlay access backbone switching network. GEO-LEO integration can mitigate network congestion by cross-migrating traffic requests to/from the GEO and LEO layers, thus improving load balancing. The bottom layer is the aerial network based on HAPs, which may connect together for a larger regional coverage. Specifically, the aerial layer may act as a relay for connections between terrestrial users and the higher satellite layers. This approach provides an additional degree of robustness in case one aerial/space platform is damaged, as other layers can temporarily serve terrestrial traffic requests. However, although the hybrid GLHE system is superior to the traditional two-tier systems, it is hard to achieve full deployment due to its high cost and management complexity, especially on the satellite layers.

\section{SYSTEM DESIGN}

In this section, we present the channel characterization for space/air communications according to the latest 3GPP and ITU guidelines (Sec. III-A), and related system parameters (Sec. III-B).

\section{A. Channel Models}

We consider a downlink system model in which GEOs, LEOs, HAPs and terrestrial base stations form a 3D multihop network. Intermediate nodes adopt a fully cooperative amplify-and-forward (AF) relay protocol. Considering a communication system in which a GEO signal propagates through $N$ hops before arriving to its destination, the Signal to Noise Ratio (SNR) $\gamma_{i, j}^{(n)}, i, j \in\{\mathrm{G}, \mathrm{L}, \mathrm{H}, \mathrm{E}\}$, experienced at the $n-$ th hop between transmitter $i$ and receiver $j$, is computed as

$$
\gamma_{i, j}^{(n)}=\mathrm{EIRP}_{i}+\frac{G_{j}}{T}-\mathrm{PL}_{i, j}+\tau_{i, j}-k-B-\mathrm{NF} . \quad[\mathrm{dB}]
$$


TABLE I: System parameters for space, air, and ground architectures [4], [12].

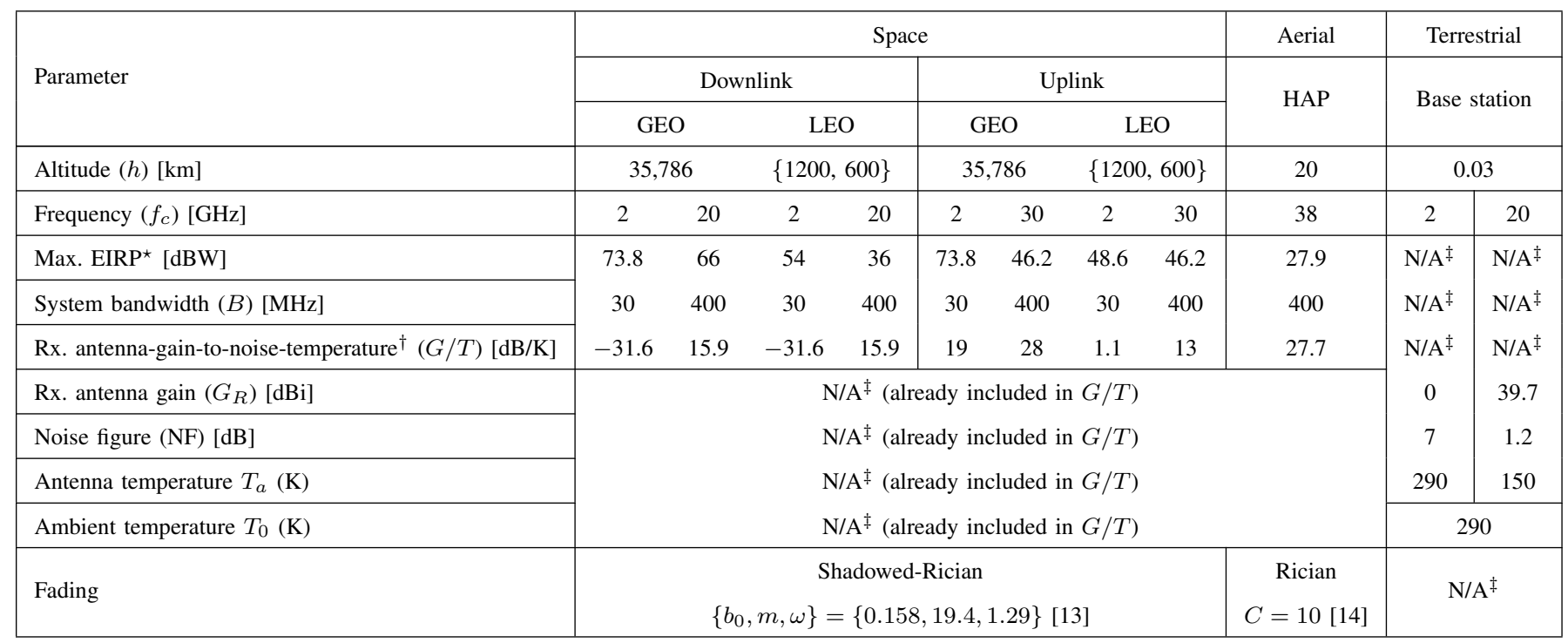

${ }_{\ddagger}$ Not available in the $3 \mathrm{GPP}$ specifications.

${ }^{\star}$ The EIRP accounts for the antenna transmit power, the cable loss, and the transmit antenna gain $\left.\mid 4\right]$.

$\dagger G / T$ accounts for the receive antenna gain, the ambient/antenna temperature, and the noise figure [4].

In Eq. (1), EIRP $i$ is the effective isotropic radiated power (which accounts for the antenna transmit power, the cable loss, and the transmit antenna gain [4]), $G_{j} / T$ is the receive antenna-gain-to-noise-temperature (which accounts for the receive antenna gain, the ambient/antenna temperature, and the noise figure), PL is the path loss, $\tau$ is the fading, $k$ is the Boltzmann constant, $B$ is the channel bandwidth, and NF is the noise figure. The end-to-end SNR for the complete AF system can then be expressed as

$$
\gamma_{\mathrm{AF}}=\left[\prod_{n=1}^{N}\left(1+\frac{1}{\gamma_{i j}^{(n)}}\right)-1\right]^{-1} .
$$

1) Path Loss: Besides free-space path loss, which increases with the carrier frequency and the propagation distance between the transmitter and the receiver, in a fully integrated space-air-ground framework the signal undergoes several other stages of attenuation due to:

- Atmospheric Gases $A_{g}$, consisting of dry air and water vapour attenuation.

- Scintillation $A_{s}$, due to fluctuations of the refractive index in the ionosphere (atmospheric turbulence scintillation in the troposphere) for below (above) $3 \mathrm{GHz}$ transmissions.

- Clutter Loss $L_{c}$, due to signal absorption and diffraction by ground objects, such as buildings or vegetation.

- Rain Absorption $A_{r}$, causing unavailability of signals due to scattering from rainfall (especially above $10 \mathrm{GHz}$ ).

It must be mentioned that, for space channels that do not involve complete penetration through the atmosphere (i.e., in the GEO-LEO, GEO-HAP, and LEO-HAP links), a simple free-space path loss model can be considered. For a more complete description of the channel model in NTN scenarios, we refer the interested reader to [9], [15].

2) Channel Fading: For the space-to-ground link, Shadowed-Rician distribution has been proposed in [13] to describe the large-scale fading due to amplitude fluctuation of the transmitted signal's envelope in the rarefied environment. In turn, a Rician distribution has been demonstrated to characterize more accurately smaller-scale fading in the air-to-ground environment [14]. As expected, space-to-space and space-to-air channels should not be affected by fading, as the density of the air in these cases is much smaller than near the ground, so many of the fading effects, such as rainfall, atmosphere, and scattering, can be neglected.

\section{B. System Parameters}

System design parameters are summarized in Table [ for both satellite- and HAP-based architectures.

1) Space Design: According to the 3GPP specifications [4], LEO stations can be deployed at an altitude of 600 or 1200 $\mathrm{km}$. Satellites can operate both in the S-bands at $2 \mathrm{GHz}$ or in the Ka-bands (i.e., within the millimeter wave (mmWave) spectrum) at 20 (30) $\mathrm{GHz}$ for downlink (uplink) transmissions, with a bandwidth of 30 and $400 \mathrm{MHz}$, respectively. It should be noticed that, while GEO satellites can support direct transmission links on the ground, thanks to their very directional and high-power transmission (up to $73.8 \mathrm{dBW}$ of EIRP in the S-bands) and large phased antennas offering fine electronic beam-steering (with a receive gain of up to 28 $\mathrm{dB} / \mathrm{K}$ in the Ka-bands), LEO orbits are typically populated by nano/picosatellites incorporating simple electronic devices to reduce component costs, and are constrained by limited power and antenna gains.

2) Aerial Design: According to the ITU guidelines [12], HAPs operate in the Ka-bands at $38 \mathrm{GHz}$ with a bandwidth of $400 \mathrm{MHz}$. While guaranteeing an ultra-flexible deployment, these elements offer an antenna-gain-to-noise-temperature of up to $27.7 \mathrm{~dB} / \mathrm{K}$ without the prohibitive costs of satellite infrastructures. 
3) Terrestrial Design: For mmWave-enabled terrestrial base stations, a receiving antenna offering a gain of $39.7 \mathrm{dBi}$ should be adopted [4], while omnidirectional unit gain should be considered at sub- $6 \mathrm{GHz}$.

\section{Multi-Layered NTNS: PERFORMANCE COMPARISON}

In this section we numerically compare the performance of the different multi-layered NTN configurations introduced in Sec. III The optimal architecture is then identified by evaluating (i) the average ergodic (Shannon) capacity, and (ii) the outage probability, i.e., the probability that the received signal on the Earth is below a predefined threshold $\epsilon$. In $\mathrm{AF}$ systems, assuming that all system parameters are independently distributed, the outage probability $\Phi_{\mathrm{AF}}$ can be calculated as

$$
\Phi_{\mathrm{AF}}=1-\left(\prod_{n=1}^{N} \mathbb{P}\left[\gamma_{i, j}^{(n)}>\epsilon\right]\right), \forall i, j \in\{\mathrm{G}, \mathrm{L}, \mathrm{H}, \mathrm{E}\} .
$$

Our analysis, which is based on the channel model described in Sec. III-A has been validated by Monte Carlo simulations obtained generating 10000 random realizations of the fading for each investigated integrated NTN configuration. Results are given as a function of the carrier frequency $f_{c}$, the signal quality threshold $\epsilon$ (varied from -20 to $40 \mathrm{~dB}$ to consider different levels of sensitivity at the receiver), the LEO altitude $h_{L}$ (which only affects those configurations in which LEO satellites are deployed, i.e., GLE and GHLE), and the elevation angle $\alpha$ between the transmitter and the receiver. Air/spaceborne stations are deployed on the same line, even though an additional average pointing loss of 0.5 $\mathrm{dB}$ is considered to characterize the loss in signal strength due to misalignment between the transmitting and receiving antennas at every hop.

Average Capacity. In Fig. 3 we compare the average capacity achieved by different NTN configurations. First, we observe that the numerical results closely follow the theoretical curves obtained applying Shannon's formula for the channel capacity, which is related to the end-to-end SNR expressed in Eq. (2) and the available bandwidth, thereby validating our framework. Second, the very large bandwidth available in the Ka-bands (Fig. 3b) delivers $10 \times$ higher capacity than in the S-bands (Fig. 3a), which are constrained by the limited frequency resources available in the 2-GHz channel. Moreover, despite the worse propagation conditions experienced at high frequency, the physical size of the antennas is so small that it becomes practical to install large antenna arrays on nonterrestrial platforms, thereby scaling up network performance by beamforming (e.g., for GEO satellites, the antenna-gain-tonoise-temperature $G / T$ is increased from -31.6 to $15.9 \mathrm{~dB} / \mathrm{K}$ at $20 \mathrm{GHz}$ ).

As expected, at $2 \mathrm{GHz}$ the standalone GE configuration shows the worst capacity performance due to the fact that atmospheric perturbations at very large distances severely deteriorate the signal's propagation without proper countermeasures. Conversely, all investigated multi-layered architectures guarantee better coverage by amplifying GEO's transmission

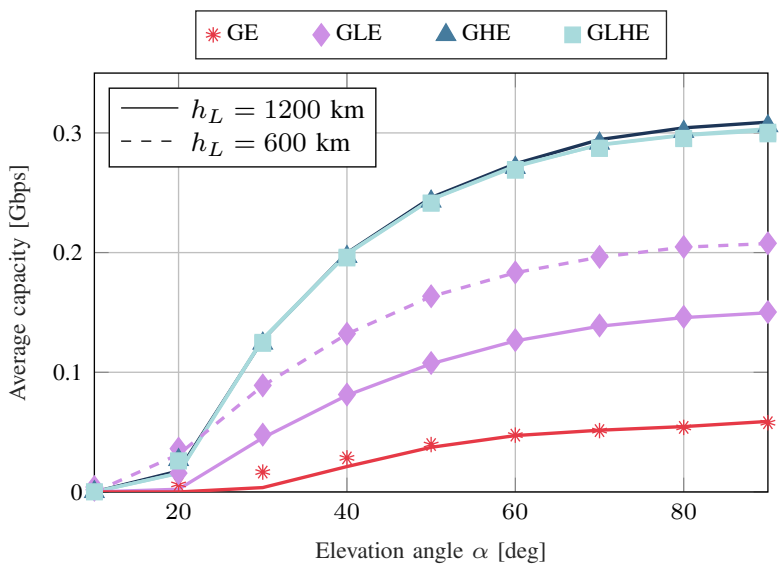

(a) $f_{c}=2 \mathrm{GHz}$

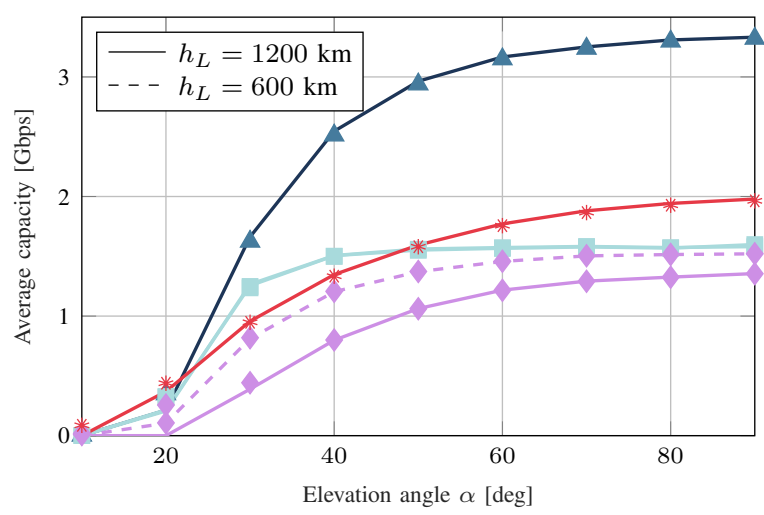

(b) $f_{c}=20$ (30) GHz for DL (UL).

Fig. 3: Average ergodic capacity vs. the elevation angle, as a function of the operational frequency for different multi-layered NTN architectures, when $\epsilon=0 \mathrm{~dB}$. The lines represent the results from the theoretical model, and the markers indicate Monte Carlo simulations.

before forwarding it to the ground. In particular, GHE offers up to $6 \times$ higher capacity than GE and emerges as the most preferable integration choice for NTNs. A fully integrated GLHE scheme achieves comparable performance but, due to the three dimensions and the mobility of the space layer, is much more complex than its GHE counterpart.

Furthermore, better capacity can be provided when a standalone GEO layer is assisted by HAPs operating in the stratosphere (in GHE) rather than by LEO satellites (in GLE). In fact, the former approach allows to decrease the length of the (bottleneck) Earth link traversing the atmosphere to only $20 \mathrm{~km}$, i.e., where HAPs are typically deployed, compared to the 600 or $1200 \mathrm{~km}$ of the latter, thus resulting in more robust channels. In any case, while 3GPP specifications [4] support two deployment options for LEOs, Fig. 3 demonstrates that it is more desirable to form a LEO constellation at $h_{L}=600$ $\mathrm{km}$ to benefit from the resulting lower path loss in the shorter space-Earth link. This, in turn, does not affect GLHE's design (which shows perfectly overlapping curves for $h_{L}=600$ and $1200 \mathrm{~km}$ ) since LEO-HAP propagation is nearly in free-space, where a difference of $600 \mathrm{~km}$ in the propagation distance accounts only for a (negligible) $6 \mathrm{~dB}$ in the overall link budget.

When transitioning to the Ka-bands, Fig. $3 \mathrm{~b}$ shows that LEO-based architectures like GLE and GLHE now are out- 


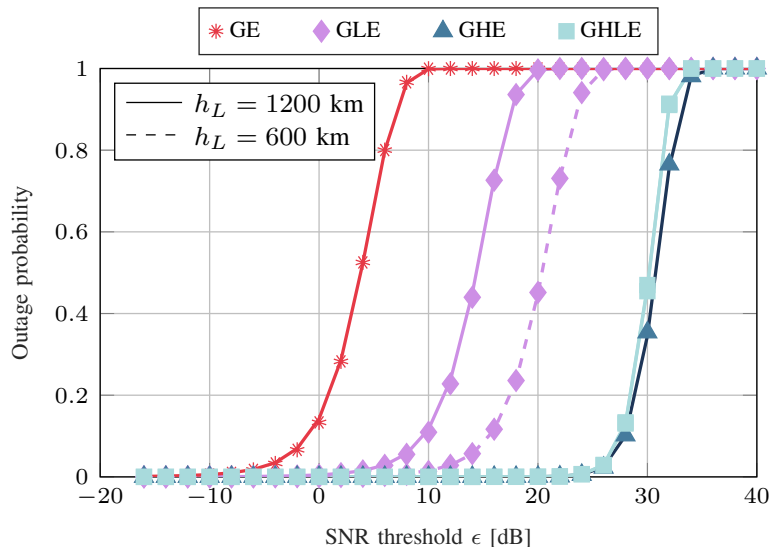

(a) $f_{c}=2 \mathrm{GHz}$.

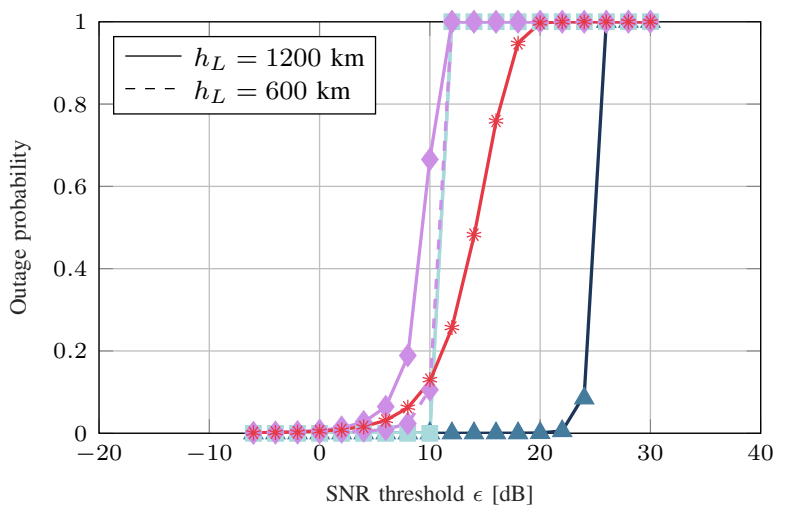

(b) $f_{c}=20(30) \mathrm{GHz}$ for $\mathrm{DL}(\mathrm{UL})$

Fig. 4: Outage probability vs. SNR threshold, as a function of the operational frequency for different multi-layered NTN architectures, when $\alpha=90 \mathrm{deg}$. The lines represent the results from the theoretical model, and the markers indicate Monte Carlo simulations.

performed even by the baseline GE configuration due to a simpler hardware implementation in LEO compared to GEO satellites: for example, Table I indicates that LEO's EIRP for relaying operations is as low as $36 \mathrm{dBW}$ at $20 \mathrm{GHz}$, compared to the $66 \mathrm{dBW}$ of GEO satellites.

Finally, Fig. 3 illustrates that the system performance decreases at low elevation due to the more serious impact of clutter and atmospheric loss, as the signal has to transit longer through the atmosphere [9]. This effect is particularly critical in the GHE and GLHE configurations where the visibility of HAPs, which fly at low altitude, is impaired for $\alpha<20 \mathrm{deg}$.

Outage Probability. Similar conclusions can be derived from Fig. 4, which reports the outage probability $\Phi_{\mathrm{AF}}$ for different values of the SNR threshold $\epsilon$ in various NTN configurations, in both S- and Ka-bands. We observe that $\Phi_{\mathrm{AF}}$, which is an indication of the quality of the communication, decreases when decreasing $\epsilon$, i.e., considering more complex (and expensive) receivers able to detect the signal in more noisy channels. Moreover, we see that integrating multiple non-terrestrial layers together offers better coverage compared to a standalone design: at $2 \mathrm{GHz}$ an outage probability lower that $1 \%$ (a critical requirement for most $5 \mathrm{G} / 6 \mathrm{G}$-oriented services) is achieved for $\epsilon=24 \mathrm{~dB}$ in the GHE structure, against $\epsilon=-6 \mathrm{~dB}$ in the $\mathrm{GE}$ case, thus ensuring more robust and continuous connectivity at lower costs. This is because the

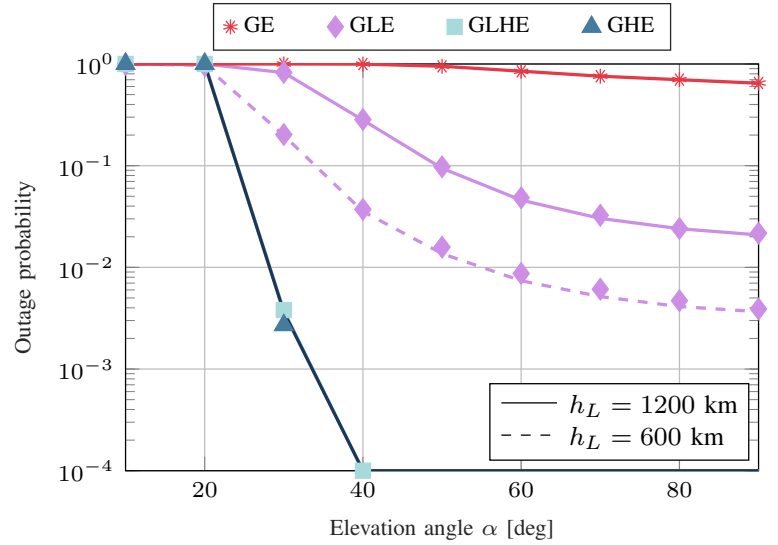

(a) $f_{c}=2 \mathrm{GHz}$.

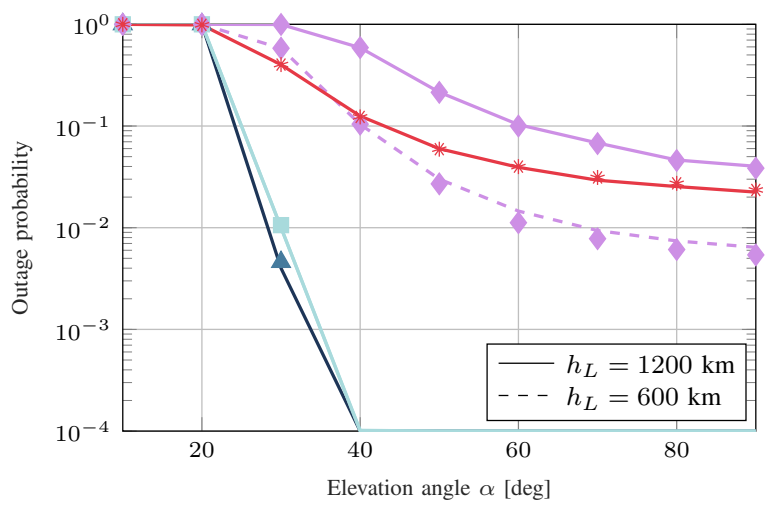

(b) $f_{c}=20(30) \mathrm{GHz}$ for DL (UL).

Fig. 5: Outage probability vs. the elevation angle, as a function of the operational frequency for different multi-layered NTN architectures, when $\epsilon=5 \mathrm{~dB}$. The lines represent the results from the theoretical model, and the markers indicate Monte Carlo simulations.

intermediate HAP permits to establish shorter-range communications in the Earth link, thus ensuring better signal quality and stronger received power. As already mentioned, LEO relays exhibit lower performance gains as channel conditions in the space layer are already sufficiently good to guarantee satisfactory signal quality even without amplification.

Furthermore, we observe that NTNs operating in the Sbands (Fig. 4a) provide more reliable communications than in the Ka-bands (Fig. $4 \mathrm{~b}$ ) since they present a lower outage probability. This is due to the increased variability of the channel conditions at $20 / 30 \mathrm{GHz}$ due to clutter loss and reflection from terrain and buildings, and the more severe impact of atmospheric absorption. Specifically, LEO-based configurations experience the most significant performance deterioration by more than $20 \mathrm{~dB}$ compared to communications in the S-bands: this is due to, besides worse propagation characteristics, the simpler hardware design in LEO satellites. The multi-layered GHE architecture, although suffering, on average, from a $5 \mathrm{~dB}$ performance loss, still stands out at the most desirable option to minimize the outage probability.

Finally, Fig. 5 illustrates that the outage performance improves consistently when increasing the elevation angle $\alpha$, as a result of the shorter path of the signal and the lower impact of scintillation. In this case, it appears evident that point-to-point GE deployments are certainly not compatible 


\begin{tabular}{|ll} 
* $\operatorname{GE}$ & $\operatorname{GHLE}$ \\
$\operatorname{GLE}\left(h_{L}=1200 \mathrm{~km}\right)$ & $\operatorname{GLE}\left(h_{L}=600 \mathrm{~km}\right)$
\end{tabular}

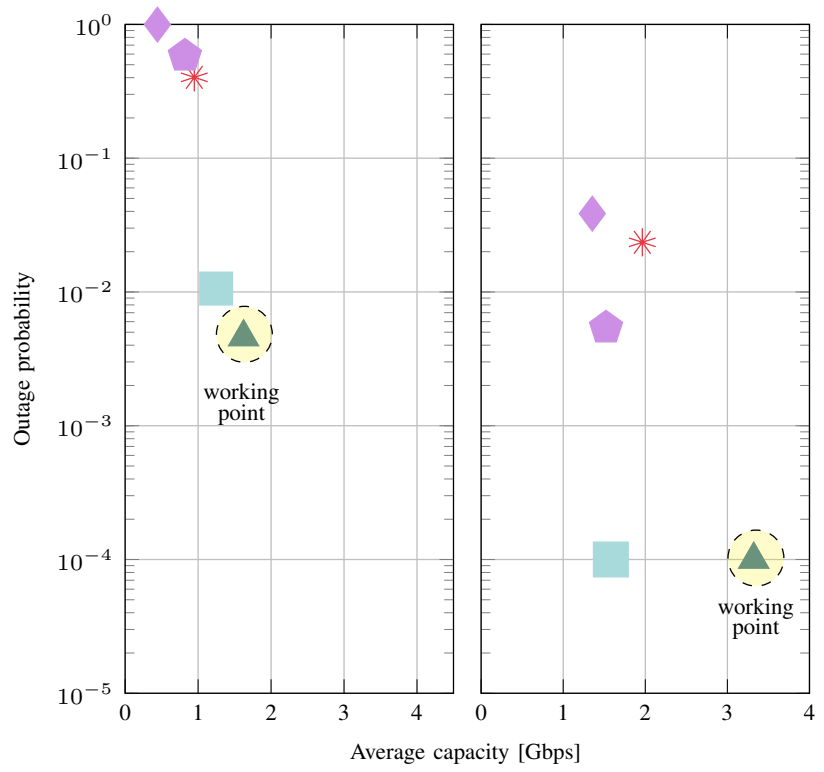

Fig. 6: Outage probability vs. average ergodic capacity trade off for different multi-layered NTN architectures. We set $\alpha=30 \mathrm{deg}$ (left), $\alpha=90 \mathrm{deg}$ (right), $\epsilon=5 \mathrm{~dB}$, and $f_{c}=20$ (30) $\mathrm{GHz}$ for DL (UL).

with robustness. In particular, the long transmission distance in the GEO-Earth link makes $\Phi_{\mathrm{AF}}$ increase above $50 \%$ for all values of $\alpha$, even when considering perfect angular alignment (i.e., $\alpha=90 \mathrm{deg}$ ). Outage is also unacceptably high for $\alpha<20 \mathrm{deg}$ in all investigated configurations, even though communication might still be established in the open (rural) environment where clutter loss is negligible. In any case, the multi-layered approach permits to support more reliable communications (with values of outage probability lower than $10^{-4}$ ) by relaying the GEO signal which would otherwise be undetectable. It should also be noticed that, while GE's performance improves when operating at $20 \mathrm{GHz}$ because of the larger antenna gains achievable by beamforming, LEO relaying can guarantee lower outage probability only when intermediate satellites are deployed at $h_{L}=600 \mathrm{~km}$, i.e., when the endpoints of the space-Earth link are progressively closer.

Comparison. To summarize the conclusions from the previous paragraphs, Fig. 6 compares the outage vs. capacity performance of different multi-layered hierarchical networks. As anticipated, the deployment of intermediate HAPs in the stratosphere (configuration GHE) ensures up to $1.75 \times$ better capacity in the Ka-bands than point-to-point GEO transmissions (configuration GE), while resulting in more robust communications. The GHE approach outperforms even the GLE configuration by 2 orders of magnitude in terms of outage probability and by more than $2 \times$ in terms of average capacity, thus making it clear that LEO satellites are not desirable to relay the upstream signal towards the ground. Furthermore, a fully integrated space-air-ground scenario (configuration GLHE) does not enhance the system performance beyond
GHE's (the GLHE capacity decreases by $42 \%$ compared to GHE), while in turn resulting in more complex and expensive network management. Finally, Fig. 6 (right) shows that better performance can be generally guaranteed at high elevation thanks to the shorter path and lower attenuation of the signal.

\section{CONCLUDing REMARKS}

$6 \mathrm{G}$ research is just in its infancy and there remain many open challenges to solve, including whether and how to design NTNs to assist terrestrial communication. In this work we addressed this issue by proposing multi-layered hierarchical networks in which the merits of the space, air, and ground layers are incorporated together to improve quality of service. Specifically, we compared the performance of different cooperative architectures against a standalone GEO constellation, and evaluated which degree of integration offers better capacity and outage probability. Our results proved that HAP relays (configuration GHE) can best bridge the satellite signal to the ground while ensuring up to $6 \times$ better capacity than point-topoint GEO transmissions. We also demonstrated that hardware constraints in the Ka-bands make LEO-based relaying configurations not desirable.

As part of our future work, we will consider end-to-end simulations to assess the benefits of the proposed multi-layered integrations in terms of network-related metrics such as the overall transmission latency, the achievable throughput, and the packet delivery rate. Moreover, we will analyze the effect of a dynamic network scenario, thereby accounting for the intrinsic mobility of LEO and HAP relays.

\section{REFERENCES}

[1] M. Giordani, M. Polese, M. Mezzavilla, S. Rangan, and M. Zorzi, "Toward 6G networks: Use cases and technologies," IEEE Communications Magazine, vol. 58, no. 3, pp. 55-61, Mar. 2020.

[2] M. Giordani and M. Zorzi, "Non-Terrestrial Networks in the 6G Era: Challenges and Opportunities," IEEE Network, vol. 35, no. 2, pp. 244 251, Mar. 2021.

[3] A. Chaoub, M. Giordani, B. Lall et al., "6G for bridging the digital divide: Wireless connectivity to remote areas," IEEE Wireless Communications Magazine, 2021.

[4] 3GPP, "Solutions for NR to support Non-Terrestrial Networks (NTN)," TR 38.821 (Release 16), 2020.

[5] E. Cianca, R. Prasad, M. De Sanctis, A. De Luise, M. Antonini, D. Teotino, and M. Ruggieri, "Integrated satellite-HAP systems," IEEE Communications Magazine, vol. 43, no. 12, pp. 33-39, Dec. 2005.

[6] J. Liu, Y. Shi, Z. M. Fadlullah, and N. Kato, "Space-air-ground integrated network: A survey," IEEE Communications Surveys Tutorials, vol. 20, no. 4, pp. 2714-2741, Fourthquarter 2018.

[7] Z. Zhou, J. Feng, C. Zhang, Z. Chang, Y. Zhang, and K. M. S. Huq, "SAGECELL: Software-defined space-air-ground integrated moving cells," IEEE Communications Magazine, vol. 56, no. 8, pp. 92-99, Aug. 2018.

[8] F. Rinaldi, H.-L. Maattanen, J. Torsner, S. Pizzi, S. Andreev, A. Iera, Y. Koucheryavy, and G. Araniti, "Non-Terrestrial Networks in 5G \& Beyond: A Survey," IEEE Access, vol. 8, pp. 165 178-165 200, Sept. 2020 .

[9] M. Giordani and M. Zorzi, "Satellite communication at millimeter waves: A key enabler of the 6G era," in International Conference on Computing, Networking and Communications (ICNC), 2020.

[10] N. Zhao, W. Lu, M. Sheng, Y. Chen, J. Tang, F. R. Yu, and K. Wong, "UAV-assisted emergency networks in disasters," IEEE Wireless Communications, vol. 26, no. 1, pp. 45-51, Feb. 2019.

[11] J. Ye, S. Dang, B. Shihada, and M.-S. Alouini, "Space-air-ground integrated networks: Outage performance analysis," IEEE Transactions on Wireless Communications, vol. 19, no. 12, pp. 7897-7912, Aug. 2020. 
[12] ITU-R, "Deployment and technical characteristics of broadband high altitude platform stations in the fixed service in the frequency bands 6 440-6 520 MHz, 21.4-22.0 GHz, 24.25-27.5 GHz, 27.9-28.2 GHz, 31.0$31.3 \mathrm{GHz}, 38.0-39.5 \mathrm{GHz}, 47.2-47.5 \mathrm{GHz}$ and $47.9-48.2 \mathrm{GHz}$ used in sharing and compatibility studies," F.2439-0, 2018.

[13] A. Abdi, W. C. Lau, M.-S. Alouini, and M. Kaveh, "A new simple model for land mobile satellite channels: First- and second-order statistics," IEEE Transactions on Wireless Communications, vol. 2, no. 3, pp. 519528, May 2003.

[14] A. Alsharoa and M.-S. Alouini, "Improvement of the global connectivity using integrated satellite-airborne-terrestrial networks with resource optimization," IEEE Transactions on Wireless Communications, vol. 19, no. 8, pp. 5088-5100, Aug. 2020.

[15] 3GPP, "Study on New Radio (NR) to support non-terrestrial networks (Release 15)," TR 38.811, 2019.

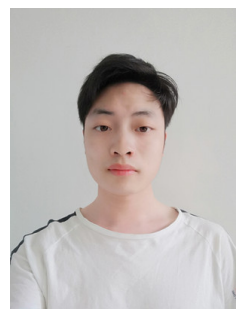

Dengke Wang was born in Sichuan, China. He received the B.Sc. degree from the University of Electronic Science and Technology of China in 2019. Now, he is pursuing the M.Sc. degree in King Abdullah University of Science and Technology (KAUST), Saudi Arabia. His main research interests include performance analysis and modeling of wireless communication networks and softwaredefined radio.

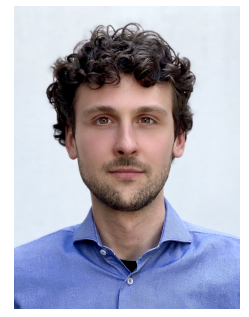

Marco Giordani [M'20] received his Ph.D. in Information Engineering in 2020 from the University of Padova, Italy, where he is now a postdoctoral researcher and adjunct professor. He visited NYU and TOYOTA Infotechnology Center, Inc., USA. In 2018 he received the "Daniel E. Noble Fellowship Award" from the IEEE Vehicular Technology Society. His research focuses on protocol design for $5 \mathrm{G} / 6 \mathrm{G}$ mmWave cellular and vehicular networks.

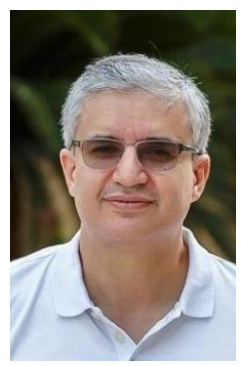

Mohamed-Slim Alouini [S'94-M'98-SM'03-F'09] was born in Tunis, Tunisia. He received the Ph.D. degree in Electrical Engineering from the California Institute of Technology (Caltech), Pasadena, CA, USA, in 1998. He served as a faculty member in the University of Minnesota, Minneapolis, MN, USA, then in the Texas A\&M University at Qatar, Education City, Doha, Qatar before joining KAUST, Thuwal, Makkah Province, Saudi Arabia as a Professor of Electrical Engineering in 2009. His current research interests include the modeling, design, and performance analysis of wireless communication systems.

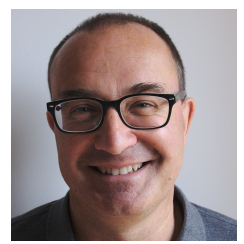

Michele Zorzi [F'07] is with the Information Engineering Department of the University of Padova, focusing on wireless communications research. He was Editor-in-Chief of IEEE Wireless Communications from 2003 to 2005, IEEE Transactions on Communications from 2008 to 2011, and IEEE Transactions on Cognitive Communications and Networking from 2014 to 2018. He served ComSoc as a Member-atLarge of the Board of Governors from 2009 to 2011, as Director of Education and Training from 2014 to 2015, and as Director of Journals from 2020 to 2021. 